\title{
Médiévales
}

Langues, Textes, Histoire

44 | printemps 2003

Le diable en procès

\section{Jean-Marie Fritz, Paysages sonores du Moyen Âge. Le} versant épistémologique

Paris, Champion, collection « Sciences, Techniques et civilisations du Moyen Âge à l'aube des Lumières ", 2000, 478 p.

\section{Christopher Lucken}

\section{(2) OpenEdition \\ Journals}

Édition électronique

URL : https://journals.openedition.org/medievales/955

DOI : $10.4000 /$ medievales.955

ISSN : $1777-5892$

Éditeur

Presses universitaires de Vincennes

\section{Édition imprimée}

Date de publication : 1 juin 2003

Pagination : 218-222

ISBN : 2-84292-142-9

ISSN : 0751-2708

Référence électronique

Christopher Lucken, " Jean-Marie Fritz, Paysages sonores du Moyen Âge. Le versant épistémologique », Médiévales [En ligne], 44 | printemps 2003, mis en ligne le 13 mars 2006, consulté le 24 avril 2022.

URL : http://journals.openedition.org/medievales/955; DOI : https://doi.org/10.4000/medievales.955

Ce document a été généré automatiquement le 24 avril 2022.

Tous droits réservés 


\section{Jean-Marie Fritz, Paysages sonores du Moyen Âge. Le versant épistémologique}

Paris, Champion, collection « Sciences, Techniques et civilisations du Moyen Âge à l'aube des Lumières ", 2000, 478 p.

\section{Christopher Lucken}

1 «Si l'homme ne retient pas les sons dans sa mémoire, ils périssent, car ils ne peuvent être écrits ", affirme Isidore de Séville dans la section de ses Étymologies consacrée à la musique - citation que l'on peut lire au seuil de l'ouvrage de J.-M. Fritz. Ce n'est que depuis la fin du xix ${ }^{\mathrm{e}}$ siècle que les sons ont la possibilité d'être mémorisés grâce à des techniques d'enregistrement qui sont venus s'ajouter à celles utilisées pour conserver les traces du passé, l'écriture et l'image principalement. Auparavant, il n'y avait pas d'autre moyen de retenir un son qui s'était produit que de s'en remettre à sa mémoire, pour autant bien sûr qu'on avait eu la chance ou la possibilité de l'entendre. Si l'on peut avoir aujourd'hui une certaine idée de l'environnement sonore $\mathrm{du} \mathrm{xx}^{\mathrm{e}}$ siècle (et encore, tout n'a pas été enregistré), comment savoir ce que les hommes d'autrefois ont entendu? Que peut-on connaître, par exemple, des productions orales et musicales des sociétés dépourvues d'écriture? Que sait-on des contes et des chants qui n'ont pas été préservés des atteintes du temps à l'aide de lettres ou de notes?

2 L'intérêt, relativement récent, pour les manifestations sonores propres à différentes périodes de l'histoire peut être rattaché aux recherches qui ont été consacrés aux phénomènes relevant de l'oralité. Divers domaines de recherche ont, pour ce qui concerne la période médiévale, inspiré tout particulièrement les travaux de $\mathrm{P}$. Zumthor qui, après avoir publié une Introduction à la poésie orale (1983), s'est consacré, dans La Poésie et la voix dans la civilisation médiévale (1984) puis dans La Lettre et la voix (1987), à la vocalité d'une « littérature » médiévale pour laquelle la voix ne serait pas seulement le support d'une transmission orale mais un élément constitutif des œuvres elles-mêmes. Cependant, c'est surtout à un musicien et compositeur canadien que l'on doit l'idée de reconstruire le paysage sonore du passé dans son ensemble: R. M. Schafer, dont le livre désormais classique, The Tuning of the World. Toward a Theory of Soundscape Design, publié 
en 1977, a été traduit en français en 1979 sous le titre suivant : Le Paysage sonore. Toute l'histoire de notre environnement sonore à travers les âges.

3 Cette sonorisation d'un monde qui semblait s'être replié en silence pour n'avoir laissé que des images adressées à la vue était susceptible de rejoindre l'intérêt que les historiens accordaient depuis plusieurs années aux mentalités et, plus particulièrement, à la vie quotidienne. Toutefois, si, par exemple, les bruits de la ville et des fêtes pouvaient faire partie d'études générales portant sur divers aspects des sociétés humaines, les historiens n'avaient guère consacré de travaux spécifiques aux phénomènes sonores produits dans le passé. C'est à A. Corbin que l'on doit plus particulièrement de s'être interrogé sur les modalités de l'attention des hommes aux sons, sur les seuils de perception et de tolérance qui peuvent caractériser différents lieux ou différents moments de l'histoire humaine, ou sur la signification sociale des manifestations sonores.

4 En 2000, J.-P. Gutton faisait paraître une synthèse sur la question, Bruits et sons dans notre histoire. Essai sur la reconstitution du paysage sonore, qui décrit les principales caractéristiques de l'environnement sonore, social et matériel, du Moyen Âge à aujourd'hui, avec ses constantes et ses nombreuses transformations. La période médiévale (à laquelle est consacrée une petite dizaine de pages) paraît partagée entre, d'une part, une présence importante de bruits de toutes sortes (en particulier dans les villes, avec ses rassemblements festifs, les cris des marchands et des artisans faisant la réclame de leurs produits dans la rue, le tapage diurne et nocturne de ses habitants, les charivaris et toutes sortes de manifestations bruyantes plus ou moins rituelles), et, d'autre part, le silence (notamment dans les monastères où les moines sont soumis à une règle qui l'exige).

5 Le paysage sonore qui résulte de la lecture du livre de J.-M. Fritz, publié la même année que ce dernier ouvrage, se présente de manière sensiblement différente. Cela n'est pas seulement dû au fait que ce livre se concentre sur le seul Moyen Âge et qu'il serait donc à même d'offrir un panorama à la fois plus varié et plus complet. C'est surtout que son objet n'est pas le même. En effet, J.-M. Fritz ne cherche pas tant à restituer le contexte sonore caractéristique de la société médiévale, même si cet aspect de la question est pris en considération. Il s'intéresse plutôt aux différents traités qui, au Moyen Âge, ont parlé du son et de différentes manifestations sonores afin de préciser la manière dont ils les décrivent et de dégager le statut particulier accordé à l'ouïe (plutôt qu'à la vue) pour une connaissance du monde et la constitution d'un savoir. Les sources employées par J.-M. Fritz ne sont donc pas les mêmes que celles que pourrait privilégier un historien. Les textes cités sont issus principalement des traditions patristique, médicale et philosophique. Ils sont écrits en latin (ou en ancien français : mais il s'agit alors, surtout, de traductions ou d'autres formes de reprises). S'y ajoutent des textes littéraires. En revanche, J.-M. Fritz renonce à utiliser les sources iconographiques et musicales. Le projet de ce livre est donc moins sociologique que, comme le souligne son sous-titre, épistémologique. En même temps que sont abordés certains phénomènes auditifs singuliers (notamment la voix humaine, les cris des animaux, la musique humaine et celle du monde), J.-M. Fritz étudie pour l'essentiel les théories qui président à l'existence même d'un horizon sonore pour la pensée médiévale; il dégage du même coup les présupposés théoriques qui sont à la fois susceptibles d'en conditionner l'écoute, d'en déterminer la nature singulière et de constituer pour nous un préalable nécessaire si l'on veut rendre compte du paysage sonore du Moyen Âge. 
6 Ce livre comprend six chapitres. Le premier concerne la place qu'occupe le sens de l'ouie dans le système des cinq sens et, plus particulièrement, les relations conflictuelles qu'il entretient avec la vue. La première partie de ce chapitre initial porte sur la primauté généralement accordée à la vue, tandis que la seconde interroge les raisons qui ont pu amener à lui préférer le sens auditif, lié à la voix, à la musique et au langage. Le deuxième chapitre est consacré, d'une part, à l'anatomie de l'oreille et du système auditif, d'autre part, à la voix et au mode de propagation des phénomènes sonores, ainsi qu'à certains phénomènes auditifs singuliers. Le troisième chapitre comporte cinq parties. Les quatre premières concernent respectivement les sons liés à chacun des quatre éléments : la terre (avec les bruits provoqués par les tremblements d'une masse chaotique en fusion qui semblent annoncer les grincements caractéristiques de l'Enfer), l'eau (avec le fracas des vagues de la mer, le murmure des sources et certains phénomènes musicaux singuliers qui lui sont associés), l'air (avec notamment le vent) et enfin le feu (avec le tonnerre et les coups de marteaux du forgeron frappant son enclume auxquels Pythagore prêtera une oreille attentive). La cinquième partie de ce chapitre complète cette description du paysage sonore et musical du monde tel qu'ont pu le concevoir (sinon à proprement parler l'entendre) les intellectuels du Moyen Âge, en traitant d'une question essentielle pour comprendre la théorie musicale issue de Pythagore comme la structure de l'univers décrite par le Timée de Platon: la musique des sphères. Le chapitre suivant porte sur les cris des animaux (auquel s'ajoute une Annexe fort utile répertoriant les textes qui les mentionnent). Il semble ainsi prendre place à l'intérieur d'un plan qui aurait été établi sur le modèle des six jours de la Création et, plus généralement, sur celui de l'histoire biblique. Le cinquième chapitre poursuit le propos du précédent en traitant plus précisément de la voix ou du chant de certains animaux dans leur rapport (analogique ou métaphorique) à la voix humaine (par exemple, le rossignol, la sirène ou le perroquet). Le dernier chapitre est consacré à la voix humaine dans son rapport au Verbe divin. La première partie l'aborde du côté de la chute (depuis la voix séductrice du serpent jusqu'à Babel); la seconde du côté de la rédemption (de l'Annonciation à la Pentecôte, en passant par la Passion et le Résurrection). Enfin, la conclusion nous mène en direction de l'Apocalypse.

7 Ce livre, extrêmement riche et stimulant, de J.-M. Fritz se propose donc de rassembler la totalité des phénomènes sensibles à l'ouïe qui relèvent du monde médiéval (un monde toutefois, on l'aura compris, qui est celui des clercs et des livres plutôt que celui dans lequel ont pu vivre les hommes du Moyen Âge). L'excellent index permettra à tous ceux qui sont intéressés par telle ou telle composante de ce paysage sonore de s'y retrouver aisément. Mais, par delà l'intérêt que présentent les différents sujets traités, une des grandes qualités de ce livre est de nous offrir une vision d'ensemble dans un domaine relativement négligé et où il ne semble pas y avoir eu jusqu'à aujourd'hui de véritable tentative de synthèse. Il associe du même coup des phénomènes qui pouvaient sembler ne pas avoir de rapport les uns avec les autres et permet de comprendre la cohérence que présentent les différents paysages sonores qui s'y trouvent décrits. Il n'est cependant pas sans susciter un certain nombre d'interrogations. Mais, étant donné l'ambition et l'ampleur de cet ouvrage, comment pouvait-il en être autrement ?

Il est par exemple tout à fait légitime d'étudier les sons des animaux décrits dans les bestiaires et la tradition encyclopédique. Mais il me semble qu'il aurait été nécessaire de souligner davantage ce parti pris, ne serait-ce que pour mieux mettre en évidence la 
spécificité de ces textes (qui ne répondent d'ailleurs pas tous au même projet). Peut-on dire que, «pour Richard de Fournival », « la belette conÿoit par l'oreille et met bas par la bouche" (p.167) ? Son Bestiaire d'Amours ne vise pas à "adapter au contexte de l'amour courtois» (p. 259) la tradition du Physiologus: son propos ne concerne pas véritablement les animaux, mais bien plutôt le chant d'amour des troubadours et des trouvères. D'ailleurs, les bestiaires ne sauraient être confondus avec des ouvrages de zoologie : aussi ne peut-on parler à leur propos de " naïveté » et affirmer qu'« en marge de la science " dont ils feraient preuve «se développe un discours plus fantaisiste où l'observation laisse la place à l'imagination » (p. 165). Ni les bestiaires ni même les encyclopédies ne consistent à rendre compte des cris des animaux que pouvaient entendre les paysans et les chasseurs du Moyen Âge. Personne n'a jamais entendu le chant des sirènes ailleurs que dans des textes (sinon de manière métaphorique). On aurait cependant souhaité que ces ouvrages soient mis en relation avec l'expérience même des hommes. Qu'en est-il, par exemple, de la beauté du chant du coq célébré par saint Ambroise pour l'oreille d'un paysan? Quelle influence est susceptible d'avoir sur son écoute sa présence au sommet des églises, où il figure le prédicateur annonçant le soleil de la résurrection à venir? J.-M. Fritz remarque les différentes transcriptions onomatopéiques de son chant dans différentes langues, notant qu'elles résultent probablement «de l'oreille de celui qui écoute et du système linguistique et phonologique dans lequel il est transcrit » (p. 200). Les conséquences de cette remarque sont essentielles et mériteraient d'être précisées. Elle nous amène en effet à questionner la nature de l'ouïe et la possibilité même que puisse exister un paysage sonore indépendamment du langage et des systèmes de représentation du monde susceptibles d'en déterminer ou d'en préparer l'écoute.

Le cuccuru latin du coq (comme le coax de la grenouille) relève de la vox inarticulata et litterata que Priscien oppose, dans son Institution grammaticale, à la fois à la vox articulata des hommes (pourvue par conséquent de signification, qu'elle soit litterata ou non) et à la vox inarticulata et illitterata à laquelle appartiennent craquements, grognements et la plupart des cris des animaux (distinctions que J.-M. Fritz mentionne p. 190). Qu'un son non scriptible, étranger à la langue et à l'écriture, soit susceptible d'être transcrit de manière codifiée (comme le chant du coq), lui permettra d'être retenu dans la mémoire et d'être transmis à la postérité (si l'on suit Isidore de Séville). Mais quelle est désormais cette voix ? La mémoire de la lettre ne se contente pas, en effet, de conserver les sons : elle donne forme à l'écoute et inscrit les phénomènes concernés dans le cadre d'un système grammatical, serait-ce sous la forme d'onomatopées ou d'interjections. Aussi peut-on se demander si certains sons que l'on entend (ou certains cris que l'on produit) ne se trouvent pas déjà dans la langue avant même d'arriver dans l'oreille ou d'être formés par la bouche. Ce qui lui est étranger relève du bruit (à quoi Aristote oppose la voix en tant qu'elle est " un son pourvu de signification ») et ne saurait être traduit au moyen de lettres. Mais qu'entend-on quand on entend un bruit ? Et comment l'écrire sans contrevenir à sa nature même ? À vouloir transcrire les sons du monde qui sont a priori non scriptibles, à tenter de restituer le paysage sonore du passé, peut-on jamais faire autre chose que de les figer dans une forme plus ou moins conventionnelle qui ne saurait donner à entendre dans leur existence même des phénomènes fondamentalement illettrés? Mais, à nouveau, qu'entend une oreille avant que le son qui la traverse ne soit transcrit? Comment sait-on ce que l'on entend s'il n'y a rien pour l'inscrire dans la mémoire (lieu par excellence du savoir pour la pensée médiévale) et permettre ainsi d'en prendre connaissance? 

comme le premier versant d'une réflexion dont le second versant, à venir, serait consacré à une esthétique, soit à "l'émotion artistique" suscitée par le son, dans la mesure où ce dernier "est une composante de la représentation" et qu'il "relève d'une poétique » (p. 8). Affirmant au terme de son Introduction que «le Son participe de la Beauté de la Création, au même titre que le Nombre et la Lumière ", J.-M. Fritz conclut que « l'Épistémologie est alors inséparable d'une Esthétique " (p. 18). En effet ! Mais pourquoi donc avoir décidé de séparer esthétique et épistémologie? Qu'entendre dès lors par esthétique? Celle-ci nous renvoie-t-elle à la conscience singulière d'un écrivain dont la sensibilité (aux sons) informe une œuvre musicale ou "littéraire " (plutôt que philosophique ou théologique) (cf. p. 321) ? Mais qu'est-ce même que la musique ou la littérature au Moyen Âge? Quel rapport entretiennent-elles avec une musique des sphères qui ne relève pas simplement de «l'imaginaire » et "de poésie plutôt que de la science », comme le laisse entendre J.-M. Fritz (p. 146). Si l'harmonie des sphères est au principe de toute création fondée sur le Nombre, celle-ci est le lieu d'un savoir qui ne concerne pas seulement les phénomènes perceptibles par les sens : il porte sur les natures de l'univers et de l'homme. J.-M. Fritz mentionne d'ailleurs les comparaisons qui remontent à l'Antiquité entre l'appareil phonatoire dont est pourvu l'être humain et un instrument de musique (notamment la lyre) (p.70 et suiv.). Pourquoi ne pas avoir profité de cette analogie pour étudier l'esthétique musicale du langage au Moyen Âge (indépendamment même de toute production musicale ou poétique) et les conséquences que cela entraîne sur la notion de vox et les conditions de sa perception?

11 Je ne voudrais pas, cependant, que ces remarques mettent en cause la qualité et la pertinence de ce livre. Elles sont suscitées par les questions qu'il pose et témoignent, je l'espère, de l'intérêt et du plaisir que m'a procuré sa lecture. Elles n'effacent évidemment pas tout ce qu'il apporte à l'étude du Moyen Âge, que l'on s'intéresse à son histoire intellectuelle, à son paysage sonore ou à ses réalisations musicales et littéraires. Si j'ai pu regretter que son versant esthétique n'ait pas été traité simultanément, je n'en attends pas moins sa sortie avec impatience. Les deux volumes une fois conjoints offriront une somme remarquable sur ce sujet novateur et contribueront très certainement à promouvoir l'utilité qu'il peut y avoir d'associer à l'étude de la littérature médiévale des réflexions théoriques issues d'autres domaines que le sien. 